\title{
New roles of filopodia and podosomes in the differentiation and fusion process of osteoclasts
}

\author{
R.L. Song ${ }^{1,2 *}$, X.Z. Liu ${ }^{1,2 *}$, J.Q. Zhu ${ }^{1,2}$, J.M. Zhang ${ }^{1,2}$, Q. Gao ${ }^{1,2}$, H.Y. Zhao ${ }^{1,2}$, \\ A.Z. Sheng ${ }^{1,2}$, Y. Yuan ${ }^{1,2}$, J.H. Gu ${ }^{1,2}$, H. Zou ${ }^{1,2}$, Q.C. Wang ${ }^{1,2}$ and Z.P. Liu ${ }^{1,2}$ \\ ${ }^{1}$ College of Veterinary Medicine, Yangzhou University, Yangzhou, China \\ ${ }^{2}$ Jiangsu Co-Innovation Center for Prevention and Control of Important \\ Animal Infectious Diseases and Zoonoses, Yangzhou, China \\ *These authors contributed equally to this study. \\ Corresponding author: Z.P. Liu \\ E-mail: liuzongping@yzu.edu.cn
}

Genet. Mol. Res. 13 (3): 4776-4787 (2014)

Received March 7, 2014

Accepted June 10, 2014

Published July 2, 2014

DOI http://dx.doi.org/10.4238/2014.July.2.7

\begin{abstract}
The cytoskeleton mediates various cellular processes such as differentiation and fusion, including in the filopodia and podosomes. However, apart from cell migration and formation of the sealing zone, little is known regarding the changes and related regulatory mechanisms of the cytoskeleton and additional roles of the filopodia and podosomes during the differentiation and fusion of osteoclasts. The cytomorphology and cytoskeleton of osteoclasts in the differentiation process were evaluated using tartrate-resistant acid phosphatase staining and immunofluorescence staining. Moreover, the expression levels of Rho GTPases and enzymes related to osteoclast differentiation and bone resorption were detected by quantitative reverse transcription-polymerase chain reaction. We detected 3 types of filopodia in osteoclast precursors and only 1 type of filopodia in undifferentiated cells. Mature osteoclasts were completely devoid of filopodia. Interestingly, cell fusion was highly specific, and the fusion
\end{abstract}


initially occurred to the filopodia. Confocal images revealed that $\mathrm{F}$-actin and microtubules significantly differed among fused cells. These results suggest that filopodia and podosomes not only play important roles in cell migration and the formation of sealing zones but also in the prefusion selectivity of 2 cells and the movement direction of the cell nucleus and cytoplasm during the fusion process. In addition, cdc $42 \mathrm{v} 1$, RhoU, and RhoF regulate the formation of 3 types of filopodia during various stages of differentiation, while Rac1, Rac2, and filament A may be associated with cell selectivity during the fusion process.

Key words: Cytoskeleton; Filopodium; Fusion; Osteoclast; Podosome; Rho GTPase

\section{INTRODUCTION}

Osteoclasts (OCs) exhibit unique bone resorption activity. Most bone-related diseases are caused by over-activity of OCs that induces an imbalance in bone remodeling, including osteoporosis, periodontal diseases, rheumatoid arthritis, multiple myeloma, and metastatic cancers (Lane and Rehman, 2002; Boyle et al., 2003; Genetos et al., 2012).

Dynamic changes in the cytoskeleton, such as cell migration and differentiation, are the basis of cell function (Janmey, 1998; Niu et al., 2012). The cytoskeleton has been increasingly recognized as the central regulator of OC function and differentiation (Jurdic et al., 2006; Vaananen and Laitala-Leinonen, 2008). Rho GTPases affect the formation and distribution of various parts of the cytoskeleton by regulating dynamic changes in the cytoskeleton. Regulated processes include the formation of filopodia (Vega and Ridley, 2007) and the formation and distribution of podosomes (Ory et al., 2008). Thus, Rho GTPases are involved in OC differentiation and bone resorption.

The OC, as a very large, multinucleated cell, can be formed by monocyte/macrophage differentiation and fusion under the stimulation of receptor activator of nuclear factor kappa-B (RANKL) and macrophage colony-stimulating factor (M-CSF) (Miyamoto, 2011). Dynamic reorganization of the cytoskeleton mediates cell fusion. In addition, the fusion of OCs is a prerequisite for the formations of the sealing zone and ruffled border. The absence of cell fusion directly leads to a severe decline in the bone resorption activity of OCs (Miyamoto, 2011). Cell fusion is thus crucial to the differentiation and maturity of OCs and to bone resorption.

In OCs, F-actin is involved in the formation of filopodia, lamellipodia, and podosomes. Previous studies showed that filopodia are sensors of chemotaxis. The filopodia projection detects changes in the environment and controls the direction of cell migration (Arjonen et al., 2011; Guiet et al., 2012). However, few studies have been published concerning the role of filopodia in OC differentiation and fusion.

In monocytes and OCs, podosomes are not only associated with cell adhesion and migration but also respond to the environment by sensing the matrix (Schachtner et al., 2013). In addition, podosomes are involved in the formation of more advanced belt-like structures, such as the sealing zone, thus providing a closed microenvironment for OC bone resorption (Jurdic et al., 2006). The formation and arrangement of podosomes are crucial for bone resorption by OCs. However, the role of podosomes in OC differentiation, particularly in cell fusion, has not been thoroughly examined. 
Previous studies primarily focused on Rho GTPase-controlled cell migration of monocytes through filopodium regulation, thus influencing fusion (Leung et al., 2010; Zhao et al., 2010; Xing et al., 2012) and manipulating the formation of the sealing zone by regulating podosome distribution, which in turn affects bone resorption (Chellaiah, 2005; Jurdic et al., 2006; Itzstein et al., 2011). However, little is known regarding the other roles of filopodia and podosomes in OC differentiation and cell fusion.

In this study, the cytomorphology, cytoskeleton, and changes in filopodia and podosomes of OCs as well as the expression of Rho GTPases were determined in the processes of cell differentiation, maturation, and fusion. The specific mechanisms and their functions in OC differentiation were also predicted.

\section{MATERIAL AND METHODS}

\section{Cell culture}

RAW264.7 cells were purchased from the Type Culture Collection of the Chinese Academy of Sciences (Shanghai, China). RAW264.7 cells were cultured at a cell density of $1562.5 \mathrm{cells} / \mathrm{cm}^{2}$ in $\alpha$-minimum essential medium with or without $50 \mathrm{ng} / \mathrm{mL} \mathrm{M-CSF}$ (Peprotech; Rocky Hill, NJ, USA) and 60 ng/mL sRANKL (Peprotech), and 10\% fetal bovine serum (Hyclone; Logan, UT, USA). Cells expressing M-CSF and RANKL were cultured for 1-6 days, whereas cells that did not express M-CSF and RANKL were cultured for 1 day. These cells were used in tartrate-resistant acid phosphatase (TRAP) staining and immunofluorescence staining. Cells with or without M-CSF and RANKL were cultured for 1-5 days, which were used for quantitative reverse transcription-polymerase chain reaction (qRT-PCR).

\section{TRAP staining}

The cells were rinsed with phosphate-buffered saline (PBS), fixed for $30 \mathrm{~s}$, and stained on culture days 1, 2, 3, 4, 5, and 6 using a TRAP staining kit (Sigma-Aldrich; St. Louis, MO, USA) according to manufacturer instructions. The morphological features of the specimens were photographed.

\section{Immunofluorescence staining (IF)}

Cells were fixed in 4\% (w/v) paraformaldehyde (Sigma-Aldrich) in PBS for $15 \mathrm{~min}$, and then washed with PBS. The cells were permeabilized in 0.2\% Triton X-100 (Amresco; Solon, OH, USA) in PBS for 10 min, washed with PBS, and blocked with $2 \%$ bovine serum albumin in PBS. Next, the cells were incubated with rhodamine-conjugated phalloidin (Invitrogen; Carlsbad, CA, USA) and diluted with 2\% bovine serum albumin in PBS for 30 min. After washing 3 times with PBS, the cells were incubated for $30 \mathrm{~min}$ with fluorescein isothiocyanate-conjugated monoclonal actin- $\alpha$-tubulin monoclonal antibody (Sigma-Aldrich) and washed with PBS. Images were obtained using a confocal laser-scanning microscope (LSM510) and analyzed with the Zen software. 


\section{RNA extraction, reverse transcription, and qRT-PCR}

From days 1-5 of osteoclastogenesis, total cellular RNA at various differentiation periods was extracted using Trizol (Invitrogen) according to manufacturer instructions. Total RNA (900 ng) was reverse-transcribed into cDNA using the PrimeScript RT reagent kit with gDNA eraser (Takara; Shiga, Japan). The primers were designed using the Primer Premier 5 software from published gene sequences (http://www.ncbi.nlm.nih.gov/) and are shown in Table 1.

Table 1. Primers used in real-time PCR analyses.

\begin{tabular}{|c|c|c|c|}
\hline Gene & Accession No. & Upstream & Downstream \\
\hline RhoA & NM_016802 & CAAGGACCAGTTCCCAGAGG & CGCAGGCGGTCATAATCTTC \\
\hline RhoB & NM_007483 & AAGTGGGTGCCCGAGGTA & CGAGGTAGTCATAGGCTTGGAT \\
\hline RhoC & NM_007484 & GGAAGACCTGCCTCCTCATT & TCCACCTGCTTGCCATCCAC \\
\hline Racl & NM_009007 & GCCTGCTCATCAGTTACACG & GACGCAATCTGTCATAATCTTC \\
\hline Rac2 & NM_009008 & TGTGATGGTGGACAGTAAGCC & GAACCACTTGGCACGGACAT \\
\hline Rac3 & NM_133223 & TGGTGAGCCCAGCCTCCTTT & CCGCAGCCGTTCAATCGTAT \\
\hline RhoG & NM_019566 & CTGCTATACAACTAACGCCTTC & GTCCCACAGGTTTAGGTTCACG \\
\hline$C d c 42 v 1$ & NM_009861 & CCTTTCTTGCTTGTTGGGAC & TGCGGCTCTTCTTCGGTT \\
\hline $\operatorname{RhoQ}(T C 10)$ & NM_145491 & TATGCTTCTCCGTGGTAAATC & ACAGTCTTCAAACCCTTCTGG \\
\hline RhoJ (TCL) & ВC05 4464 & CTGTCGTAAACCCAGCCTCTT & CAGGGCAGAACATTCCAAGTAG \\
\hline $\operatorname{RhoV}(\mathrm{Chp} / \operatorname{Wrch} 2)$ & NM_145530 & GCAGCCTCATCGTCAGCTACAC & GAAGCAAGCCAGAAAGACATCG \\
\hline RhoU (Wrch1) & NM_133955 & GAGAAGCCGGTGCCTGAAGA & GCTGGGAGTCTGAGTGCTGGAT \\
\hline Rndl & NM_172612 & AGTGGTGGTCAGGTGTAAGC & GCACATAGGTCTCGGGATAG \\
\hline Rnd2 & NM_009708 & GGTGCTGGTTGGCTGTAAG & CAGAAGATCGGGAGGAACA \\
\hline Rnd3 (RhoE) & NM_028810 & GATAGTAGTAGTGGGCGACAGC & ATTCTCAAACACCGTAGGGAC \\
\hline RhoD & NM_007485 & AGATGACTATGACCGCCTCCG & ACCAGCACCTTGTCCTTACGC \\
\hline RhoF (Rif) & NM_175092 & CCCGTCGGTGTTTGAGAAGTA & GAGGACGTTGTCGTAACTGGTG \\
\hline RhoH $(T T F)$ & NM_001081105 & ATCAATGCCATAGAAGGGAAGA & GTTCGGACAGCACATTCAAATA \\
\hline RhoBTB1 & NM_001252638 & AAGGAGGAAGACCACTACCAG & GGACGAATGCCAGAAACAC \\
\hline RhoBTB2 & NM_-153514 & GGAGACCACCACAAAGACCG & CAGCCCACCAGAATGACAGG \\
\hline TRAP & NM_001102405 & GGCTACTTGCGGTTTCACTAT & TCCTTGGGAGGCTGGTCT \\
\hline FLNA & NM_010227 & GCCTACTCACAGCCACTG & GCATCACCTATCTCCGACT \\
\hline CTSK & NM_007802 & GAAGAAGACTCACCAGAAGCAG & TCCAGGTTATGGGCAGAGATT \\
\hline DC-STAMP & NM_029422 & TACGTGGAGAGAAGCAAGGAA & ACACTGAGACGTGGTTTAGGAAT \\
\hline GAPDH & GU214026 & ATGGTGAAGGTCGGTGTG & TGAAGGGGTCGTTGATGG \\
\hline
\end{tabular}

The expression levels of all genes were measured using a real-time PCR system (Applied Biosystems; Foster City, CA, USA). qRT-PCR was performed in $20-\mu \mathrm{L}$ reactions containing $200 \mathrm{ng}$ cDNA. A SYBER Premix Taq ${ }^{\mathrm{TM}}$ II Kit (Takara) was used following the recommended protocol for real-time PCR. mRNA levels were normalized to glyceraldehyde3-phosphate dehydrogenase gene transcript levels.

\section{Statistical analysis}

In this study, each reaction was carried out in triplicate. mRNA expression data were analyzed by comparing $2^{-\Delta \Delta C \mathrm{CT}}$ values. The percentage of total Rho GTPase expression was calculated as the sum of all $2^{-\Delta \Delta C T}$ values of Rho GTPases. The results are reported statistically as means \pm standard deviation. Significance was assessed by one-way analysis of variance. The results were compared between groups using analysis of variance and least significant difference post-hoc tests following appropriate transformation to achieve normalized data and equalized variance where necessary. Statistical analysis was performed using SAS 9.1.3 (SAS; Cary, NC, USA); P $<0.05$ and $\mathrm{P}<0.01$ were considered to indicate significance and high significance, respectively. 


\section{RESULTS}

\section{Changes in morphology, TRAP activity, and cytoskeletal structure during OC formation and maturation}

As shown in Figure 1a, TRAP-positive cells were first observed on day 2 of induction. A large number of OCs suddenly appeared on day 4, whereas only a minimal number appeared on day 3 . The percentage and volume of TRAP-positive cells increased significantly with increasing induction time. The cells induced on day 1 were notably more dispersed than undifferentiated cells and those induced on other days. However, the concentration was significantly different between induced and uninduced cells. Induced cells exhibited a cell-celldependent concentration, whereas uninduced cells exhibited a relatively cell-cell-independent concentration. Furthermore, the cells displayed typical dendrite-like extensions with numerous filopodia and lamellipodia on day 4. In contrast, cell edges were smooth, without filopodia and lamellipodia, on days 5 and 6. The results were confirmed by IF staining (Figure 1a, F-actin).

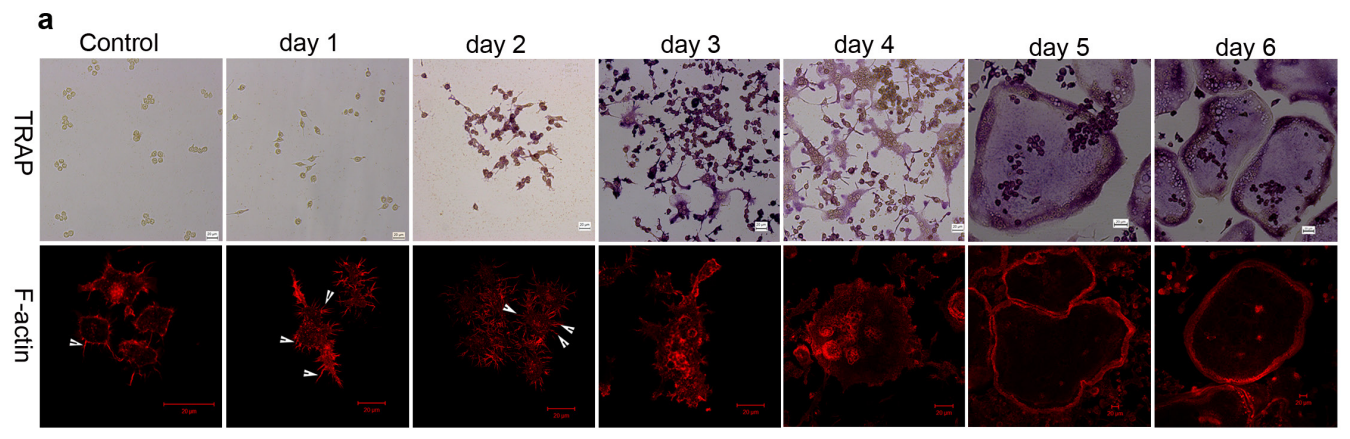

b

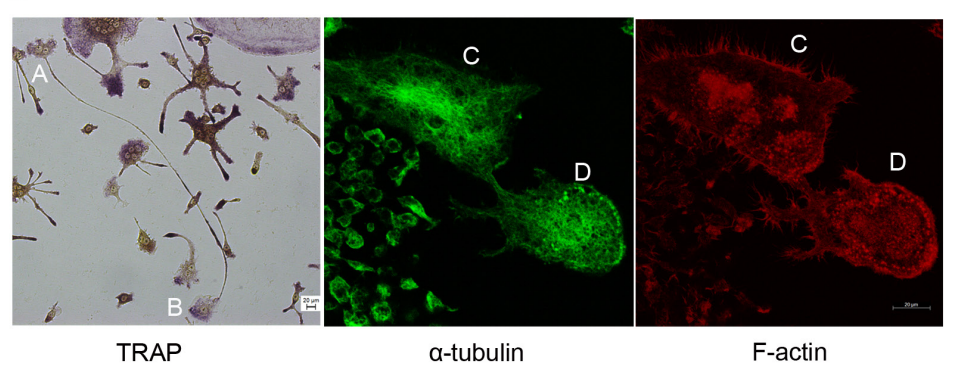

Figure 1. Morphology, TRAP activity, and cytoskeleton in OC differentiation and cell-cell fusion. (a, TRAP) To investigate changes in morphology and TRAP activity by TRAP staining, images were obtained using a Leica microscope, which showed morphology and TRAP activity changes during OC differentiation from RAW264.7 cells. (a, F-actin) Cells were stained for F-actin using rhodamine-conjugated phalloidin. (b) Cell-cell fusion in OC differentiation. TRAP staining analysis of cell-cell fusion morphology. Immunofluorescence staining (IF) analysis of cytoskeletons of fusing cells, including F-actin and $\alpha$-tubulin. IF images were obtained by confocal microscopy and analyzed using the Zen software. Confocal images show cytoskeleton dynamics at various stages of OC differentiation and fusion. 
To investigate changes in cytoskeletal structure at different stages of OC formation and maturation, cytoskeletons were analyzed by confocal microscopy, followed by IF staining for F-actin.

As shown in Figure 1a (TRAP), the process notably increased in filopodia during OC formation (days 0-4), and then disappeared during OC maturation (days 5-6). As shown in Figure 1a (F-actin), three types of filopodia were observed on OC precursors, whereas only 1 type was observed on uninduced cells. The arrangement of podosomes also underwent significant changes. Few podosomes were observed on uninduced cells, but their number and density increased with OC formation and maturation. Podosomes appeared as dots on OC precursors from days 1-3. However, some podosomes initially grouped into clusters, assembled into small rings, and eventually into a podosome belt on days 5-6.

\section{Cell-cell fusion}

As shown in Figure 1b, A and B consistently selected each other, even if they were surrounded by various OCs and OC precursors. Moreover, mutual filopodia fusion preceded cell fusion (Figure 1b, TRAP). Confocal images also revealed that F-actin and microtubules were significantly different among fused cells (Figure 1b, F-actin and $\alpha$-tubulin). For example, podosomes were grouped into clusters, while microtubules were filamentous in cell C. However, podosomes were distributed uniformly into a podosome belt at the cell edge, while microtubules were reticular in cell D. The results suggested that cell-cell fusion was selective rather than based on proximity during osteoclastogenesis and that filopodia mediated the processes of monocyte fusion.

\section{Expression of TRAP, cathepsin K, dendritic cell-specific transmembrane protein (DC-STAMP), and filamin A (FLNA)}

Expression of TRAP, cathepsin K, DC-STAMP, and FLNA was analyzed by qRT-PCR at various stages of cell differentiation. As shown in Figure 2, in the induced group, TRAP, CTSK, DC-STAMP, and FLNA expression significantly increased with increasing induction time. Compared with the uninduced group, TRAP, CTSK, and DC-STAMP levels increased, while the FLNA level decreased in the induced group. Furthermore, the TRAP level in cells induced for 2 days suddenly increased and was 68 -fold higher than that in the uninduced group. The TRAP level increased again as verified by TRAP-stained images. Similarly, the cathepsin $\mathrm{K}$ level suddenly increased on day 3 of induction and was 1600 -fold higher than that in the uninduced group. These results, as well as those of TRAP staining, demonstrated that on the third day, under the action of DC-STAMP, cells begin to fuse and very large OCs were generated by the next day. At the same time, cathepsin K expression increased rapidly, marking the start of the synthesis of enzymes relevant to bone resorption.

\section{Rho GTPase expression in the differentiation of OCs}

In Figure 3, it can be seen that the 4 genes with the highest expression in the uninduced cells and OC precursors were Rac1, cdc42V1, RhoA, and Rac2 (Figure 3, days 0-3). In OCs, the sequence was $R a c 2, R a c 1, c d c 42 v 1$, and $R h o A$ (Figure 3, days 4-5). Interestingly, Racl 
and $c d c 42 v 1$ showed identical expression levels during the entire differentiation process. Furthermore, RhoH, RhoJ, and Rnd3 were not expressed during the entire differentiation process.

\section{TRAP}

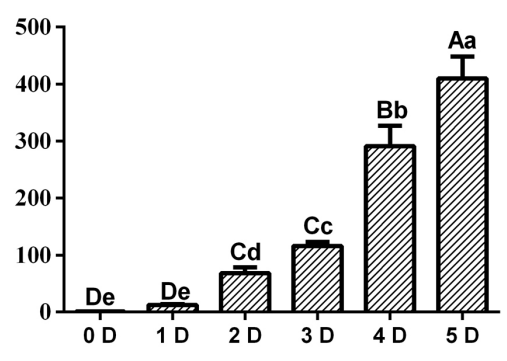

DC-STAMP

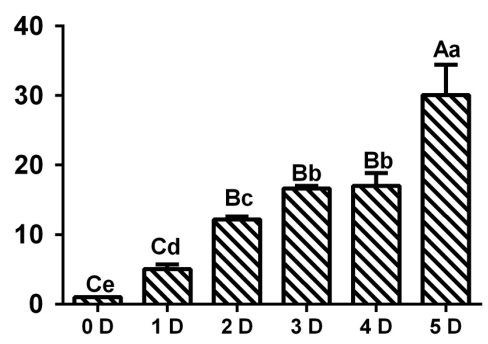

\section{cathepsin $\mathrm{k}$}

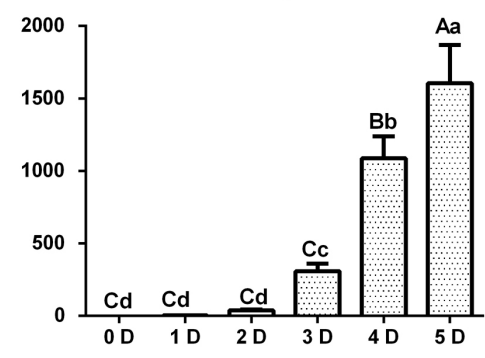

FLNA

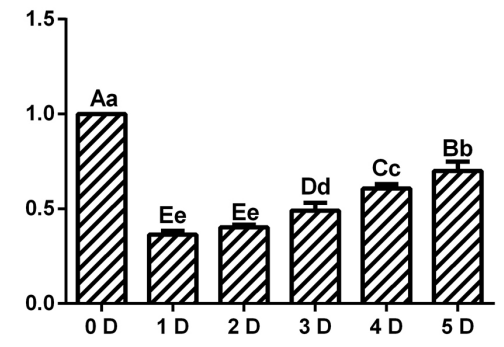

Figure 2. Expression of TRAP, CTSK, DC-STAMP, and FLNA in osteoclasts (OC) differentiation. All graphs show the cDNA levels of the indicated genes relative to GAPDH in the same sample during OC differentiation and analyzed by qRTPCR. Error bars $=95 \%$ confidence limits. Means denoted with the same letter were not significantly different. $\mathrm{D}=$ days.

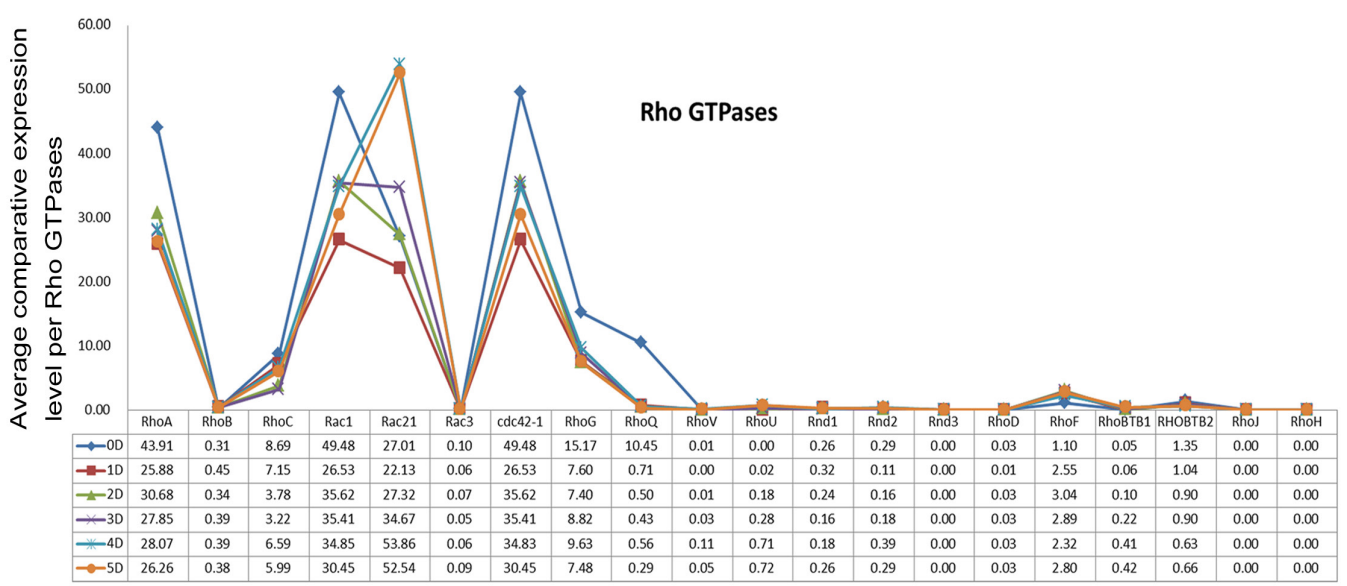

Figure 3. General expression pattern of Rho GTPases in osteoclasts differentiation. The mRNA level of Rho GTPases are shown as the average $2^{-\Delta \Delta C T}$ value per Rho GTPases of cells each day. The same lowercase letters and uppercase letters indicate an insignificant difference between groups. Different lowercase letters and uppercase letters were considered to indicate significance $(\mathrm{P}<0.05)$ and high significance $(\mathrm{P}<0.01)$, respectively. 
Compared with the genes of uninduced cells, those of Rho GTPases showed significantly decreased expression on the first day, except RhoB, Rndl, RhoU, RhoF, and RhoBTB1. Among these, expression levels of $R h o U, R h o F$, and $R h o B T B 1$ were significantly upregulated (Figure 4). This finding has not been reported in previous studies, indicating that the first day of OC differentiation is distinctive in that the cytoskeleton stability rapidly decreased. This is the first step of cytoskeleton reorganization.

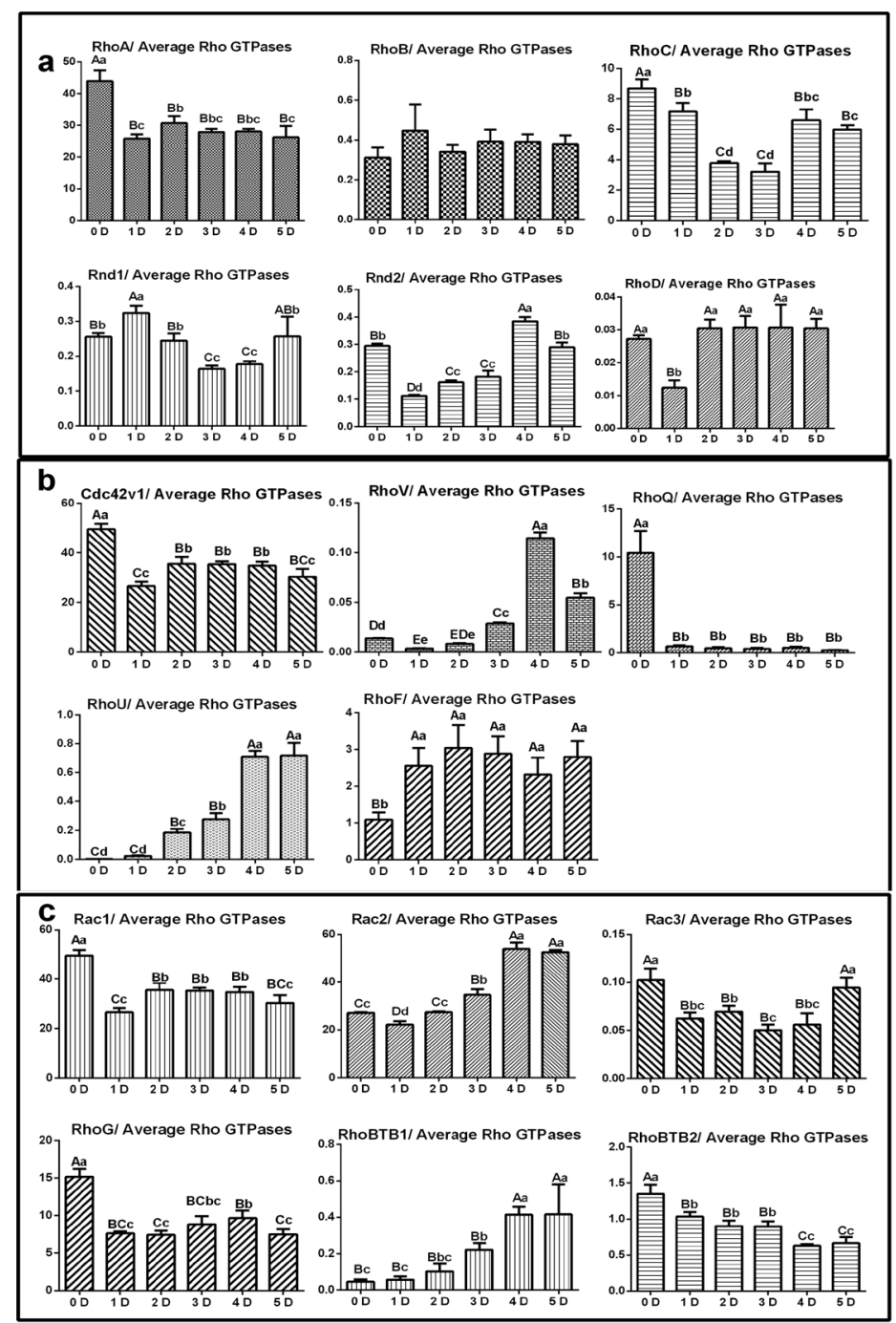

Figure 4. Comparative expression levels of Rho GTPases during osteoclast differentiation. Means with the same letter were not significantly different. Error bars: $95 \%$ confidence limits. a. Expression of the Rho subfamily, Rnd subfamily, and RhoD genes; b. expression of the $c d c 42$ subfamily and $R h o F$ genes; and c. expression of the Rac and Rho BTB gene subfamilies. The same lowercase letters and uppercase letters indicate an insignificant difference between groups. Different lowercase letters and uppercase letters were considered to indicate significance $(\mathrm{P}<$ $0.05)$ and high significance $(\mathrm{P}<0.01)$, respectively. $\mathrm{D}=$ days. 
From the first day to the fourth day of OC formation, Rnd2, RhoV, RhoU, Rac2, and RhoBTB1 showed continuously increasing expression. Except for a transient increase in $R h o D$, $c d c 42 v 1$, and Racl expression on the first day, the expression levels showed steady increases. In contrast, RhoBTB2 expression was inhibited continuously. There was no significant change in $R h o B$ expression, while $R h o Q$ expression was largely inhibited. Continuous downregulation of $R h o C$ and Rndl expression was observed in OC precursors. (Figure 4, days 1-3).

The OC maturation process occurred on days 4 and 5. Expression levels of Rnd1 and $R a c 3$ were upregulated, while those of Rnd2, $c d c 42 v 1$, RhoG, and Racl were slightly downregulated. RhoV expression was significantly downregulated. Expression of other Rho GTPases was maintained at the levels observed when OCs were produced in large quantities. (Figure 4, days 4-5).

\section{DISCUSSION}

The cytoskeleton plays an important role in OC differentiation, maturation, and fusion. However, few systematic studies on the cytoskeleton and Rho GTPases have been reported. In this study, we found that filopodia, podosomes, and Rho GTPases underwent drastic and regular variations during the $\mathrm{OC}$ differentiation, maturation, and fusion processes. The mechanisms of variation and their functions in these processes were analyzed.

\section{OC differentiation and expression of specific enzymes}

TRAP and cathepsin $\mathrm{K}$ are associated with OC formation and bone resorption, respectively (Wittrant et al., 2002). DC-STAMP is a key factor in OC fusion (Yagi et al., 2005). Variations in the expressions of these 3 genes demonstrated that the OCs had a constantly distinctive differentiation with increased culture time. This was consistent with the results of TRAP staining and IF staining.

FLNA is an actin-bound protein that forms a larger network with F-actin through cross-linking interactions (Stossel et al., 2001; Popowicz et al., 2006; Leung et al., 2010). The Rho GTPases are largely concentrated in the filopodia and function in cascade amplification by FLNA (Leung et al., 2010). Most Rho GTPases and FLNA showed reduced expression on the first day of induction, indicating a decline in cytoskeleton stability. This is the first step of cytoskeleton reorganization, which is followed by OC differentiation and maturation.

Our results showed that the differentiation and maturation process of OCs was divided into 4 stages, during which dramatic changes occurred to the cytoskeleton, cytomorphology, and relevant regulatory factors: 1 ) induced differentiation of RAW264.7 cells into OC precursors, i.e., days $0-1$; 2) incubation period of OC precursors, i.e., days $1-3 ; 3$ ) transformation of OC precursors into immature OCs, i.e., days 3-4; and 4) maturity of OCs, i.e., days 4-6. Interestingly, changes in the filopodia and podosomes appeared to occur in the cytoskeleton during the cell fusion process.

\section{OC fusion process}

The OC is formed by monocyte/macrophage fusion and has no proliferating ability (Vaananen and Laitala-Leinonen, 2008; Miyamoto, 2011). Cell fusion plays a decisive role in 
OC differentiation and bone resorption. Furthermore, most previous studies examining filopodia have concentrated on the role of filopodia in cell migration, but have ignored its role in cell fusion. Cell fusion is a highly specific process that is not affected by the proximity principle. The fusion first occurs to a portion of the filopodia. Previous reports have shown that FLNA, Rac1, and Rac2 are related to the OC chemotaxis during differentiation (Wang et al., 2008; Leung et al., 2010; Itokowa et al., 2011; Sun et al., 2013). FLNA allows Rho GTPases to accumulate at the front of filopodia, and a cascade amplifies the effect to Rho GTPases (Nobes and Hall, 1995; Ohta et al., 2006; Leung et al., 2010). In this study, Racl and Rac2 were very highly expressed. Rac2 was consistently upregulated during the entire process, which was consistent with the cell fusion process. These results indicate that Rac1, Rac2, and FLNA interact and play an important role in fusion selectivity through the action of filopodia.

\section{Changes and adjustment of filopodia}

Cell fusion and migration are crucial for OC differentiation and maturation (Leung et al., 2010; Miyamoto, 2011). Remarkably, there is only 1 type of filopodium present in undifferentiated cells. However, in OCs and OC precursors, there are 3 types of filopodia. The filopodia disappeared after sealing zone formation in mature OCs. With the increase in filopodia, the OCs and OC precursors became more scattered.

According to some previous reports, filopodia formation is regulated by the cdc 42 subfamily, which includes cde42, RhoU, RhoF, RhoV, RhoQ, and RhoJ (Nobes and Hall, 1995; Pellegrin and Mellor, 2005; Vega and Ridley, 2007; Ruusala and Aspenstrom, 2008). In our study, RhoJ was not expressed. RhoQ and $R h o J$ expression was significantly inhibited in OC precursors. Expression levels of $R h O F$ and $R h o U$ were significantly upregulated by induction. Some reports indicated that RhoF was involved in the formation of cdc42-independent filopodia (Ellis and Mellor, 2000; Pellegrin and Mellor, 2005). As an atypical Rho GTPase, RhoU shows rapid GTP exchange activity (Saras et al., 2004). Therefore, RhoU rapidly regulates filopodia formation. Although $c d c 42 v 1$ expression was downregulated under induction, it maintained the highest expression level among all Rho GTPases studied. The same type of filopodia was found in OC precursors and OCs as in the undifferentiated cells. Thus, cdc42, RhoU, and RhoF regulate the formation of 3 types of filopodia, respectively.

Based on the changes of filopodia, increased intercellular dispersion and variation of Rho GTPase expression showed that the formation of large quantities of filopodia was promoted in OC precursors and OCs through the regulation of cdc42, RhoU, and RhoF expression. As a result, the cell migration rate and mutual response of fused cells were improved, and the rate of mutual selection and approach increased. This resulted in the acceleration of cell fusion. Because of a specific mechanism, the filopodia disappeared in mature OCs, which may be conducive to increasing the strength of adhesion between OCs and the bone matrix and to facilitate the bone resorption activity of OCs.

\section{Podosomes play an important role in cell fusion and sealing zone formation}

The sealing zone is unique to OCs and provides a closed microenvironment for OC bone resorption. The protons and lytic enzymes released from cells accumulate in the sealing zone to degrade the bone matrix (Nesbitt and Horton, 1997; Luxenburg et al., 2007). The sealing zone 
is a belt-like structure formed by the compact arrangement of podosomes. Our results showed that podosomes also underwent drastic changes. The number of podosomes increased from undifferentiated cells to mature cells. The podosomes showed a change from spot-like patterns to cluster patterns, and finally to belt patterns. Such changes are significant for OC bone resorption.

Even more remarkably, differences in the arrangement pattern of podosomes between the 2 fused cells may account for the difference in the movement direction of nuclei and cytoplasma. Thus, podosomes not only play an important role in OC bone resorption but also in cell fusion, as observed in this study.

In myeloid cells, including monocytes, macrophages, and OCs, podosomes are produced to replace stress fibers and focal adhesions. The podosome, focal adhesions, and stress fibers contain some common factors (Block et al., 2008). These processes may be also regulated by Rho GTPases that control for stress fibers and focal adhesions. Subfamilies such as Rho, Rnd, Rac, and cdc42 showed regular variations in OC differentiation and maturation. Very few reports have been published regarding the role of Rho GTPases in the formation and arrangement of podosomes. RhoA (Zhang et al., 1995), cdc42 (Linder et al., 1999; Chellaiah, 2005), and Rac1 (Ory et al., 2000) were confirmed to function in the formation of podosomes. Interestingly, there was similar variation in the expression levels of these proteins. Expression of Racl and $c d c 42 \mathrm{VI}$ was identical during the entire differentiation and maturity processes.

In summary, we conducted a detailed analysis of OC differentiation, maturation, and fusion from the perspective of the cytoskeleton. We identified new roles for filopodia and podosomes during the differentiation and fusion processes of OCs. Cell fusion is highly selective; filopodia and podosomes not only play important roles in cell migration and formation of the sealing zone related to bone resorption, respectively, but also in the OC fusion process. Moreover, Rho GTPases, which are important for cytoskeleton regulation, were also analyzed with respect to their functions in $\mathrm{OC}$ differentiation and maturation. This study provides valuable information regarding osteoclast differentiation and bone resorption mechanisms.

\section{ACKNOWLEDGMENTS}

Research supported by the National Natural Science Foundation of China (\#31172373, \#31302154 and \#31372495) and the Specialized Research Fund for the Doctoral Program of Higher Education (\#20113250110003) and a project funded by the Priority Academic Program Development of Jiangsu Higher Education Institutions and the Graduate Innovation Project of Jiangsu Province (\#CXZZ12_0917). The funders had no role in study design, data collection and analysis, decision to publish, or preparation of the manuscript.

\section{REFERENCES}

Arjonen A, Kaukonen R and Ivaska J (2011). Filopodia and adhesion in cancer cell motility. Cell Adh. Migr. 5: 421-430.

Block MR, Badowski C, Millon-Fremillon A, Bouvard D, et al. (2008). Podosome-type adhesions and focal adhesions, so alike yet so different. Eur. J. Cell Biol. 87: 491-506.

Boyle WJ, Simonet WS and Lacey DL (2003). Osteoclast differentiation and activation. Nature 423: 337-342.

Chellaiah MA (2005). Regulation of actin ring formation by rho GTPases in osteoclasts. J. Biol. Chem. 280: 32930-32943.

Ellis S and Mellor H (2000). The novel Rho-family GTPase rif regulates coordinated actin-based membrane rearrangements. Curr. Biol. 10: 1387-1390.

Genetos DC, Zhou Z, Li Z and Donahue HJ (2012). Age-related changes in gap junctional intercellular communication in osteoblastic cells. J. Orthop. Res. 30: 1979-1984. 
Guiet R, Verollet C, Lamsoul I, Cougoule C, et al. (2012). Macrophage mesenchymal migration requires podosome stabilization by filamin A. J. Biol. Chem. 287: 13051-13062.

Itokowa T, Zhu ML, Troiano N, Bian J, et al. (2011). Osteoclasts lacking Rac2 have defective chemotaxis and resorptive activity. Calcif. Tissue Int. 88: 75-86.

Itzstein C, Coxon FP and Rogers MJ (2011). The regulation of osteoclast function and bone resorption by small GTPases. Small GTPases 2: 117-130.

Janmey PA (1998). The cytoskeleton and cell signaling: component localization and mechanical coupling. Physiol. Rev. 78: 763-781.

Jurdic P, Saltel F, Chabadel A and Destaing O (2006). Podosome and sealing zone: specificity of the osteoclast model. Eur. J. Cell Biol. 85: 195-202.

Lane NE and Rehman Q (2002). Osteoporosis in the rheumatic disease patient. Lupus 11: 675-679.

Leung R, Wang Y, Cuddy K, Sun C, et al. (2010). Filamin A regulates monocyte migration through Rho small GTPases during osteoclastogenesis. J. Bone Miner. Res. 25: 1077-1091.

Linder S, Nelson D, Weiss M and Aepfelbacher M (1999). Wiskott-Aldrich syndrome protein regulates podosomes in primary human macrophages. Proc. Natl. Acad. Sci. U. S. A. 96: 9648-9653.

Luxenburg C, Geblinger D, Klein E, Anderson K, et al. (2007). The architecture of the adhesive apparatus of cultured osteoclasts: from podosome formation to sealing zone assembly. PLoS One 2: e179.

Miyamoto T (2011). Regulators of osteoclast differentiation and cell-cell fusion. Keio J. Med. 60: 101-105.

Nesbitt SA and Horton MA (1997). Trafficking of matrix collagens through bone-resorbing osteoclasts. Science 276: 266-269.

Niu J, Mo Q, Wang H, Li M, et al. (2012). Invasion inhibition by a MEK inhibitor correlates with the actin-based cytoskeleton in lung cancer A549 cells. Biochem. Biophys Res. Commun. 422: 80-84.

Nobes CD and Hall A (1995). Rho, rac, and cdc42 GTPases regulate the assembly of multimolecular focal complexes associated with actin stress fibers, lamellipodia, and filopodia. Cell 81: 53-62.

Ohta Y, Hartwig JH and Stossel TP (2006). FilGAP, a Rho- and ROCK-regulated GAP for Rac binds filamin A to control actin remodelling. Nat. Cell Biol. 8: 803-814.

Ory S, Munari-Silem Y, Fort P and Jurdic P (2000). Rho and Rac exert antagonistic functions on spreading of macrophagederived multinucleated cells and are not required for actin fiber formation. J. Cell Sci. 113 (Pt 7): 1177-1188.

Ory S, Brazier H, Pawlak G and Blangy A (2008). Rho GTPases in osteoclasts: orchestrators of podosome arrangement. Eur. J. Cell Biol. 87: 469-477.

Pellegrin S and Mellor H (2005). The Rho family GTPase Rif induces filopodia through mDia2. Curr. Biol. 15: 129-133.

Popowicz GM, Schleicher M, Noegel AA and Holak TA (2006). Filamins: promiscuous organizers of the cytoskeleton. Trends Biochem. Sci. 31: 411-419.

Ruusala A and Aspenstrom P (2008). The atypical Rho GTPase Wrch1 collaborates with the nonreceptor tyrosine kinases Pyk2 and Src in regulating cytoskeletal dynamics. Mol. Cell Biol. 28: 1802-1814.

Saras J, Wollberg P and Aspenstrom P (2004). Wrch1 is a GTPase-deficient Cdc42-like protein with unusual binding characteristics and cellular effects. Exp. Cell Res. 299: 356-369.

Schachtner H, Calaminus SD, Thomas SG and Machesky LM (2013). Podosomes in adhesion, migration, mechanosensing and matrix remodeling. Cytoskeleton 70: 572-589.

Stossel TP, Condeelis J, Cooley L, Hartwig JH, et al. (2001). Filamins as integrators of cell mechanics and signalling. Nat. Rev. Mol. Cell Biol. 2: 138-145.

Sun C, Forster C, Nakamura F and Glogauer M (2013). Filamin-A regulates neutrophil uropod retraction through RhoA during chemotaxis. PLoS One 8: e79009.

Vaananen HK and Laitala-Leinonen T (2008). Osteoclast lineage and function. Arch. Biochem. Biophys 473: 132-138.

Vega FM and Ridley AJ (2007). SnapShot: Rho family GTPases. Cell 129: 1430.

Wang Y, Lebowitz D, Sun C, Thang H, et al. (2008). Identifying the relative contributions of Rac1 and Rac2 to osteoclastogenesis. J. Bone Miner. Res. 23: 260-270.

Wittrant Y, Couillaud S, Theoleyre S, Dunstan C, et al. (2002). Osteoprotegerin differentially regulates protease expression in osteoclast cultures. Biochem. Biophys. Res. Commun. 293: 38-44.

Xing L, Xiu Y and Boyce BF (2012). Osteoclast fusion and regulation by RANKL-dependent and independent factors. World J. Orthop. 3: 212-222.

Yagi M, Miyamoto T, Sawatani Y, Iwamoto K, et al. (2005). DC-STAMP is essential for cell-cell fusion in osteoclasts and foreign body giant cells. J. Exp. Med. 202: 345-351.

Zhang D, Udagawa N, Nakamura I, Murakami H, et al. (1995). The small GTP-binding protein, rho p21, is involved in bone resorption by regulating cytoskeletal organization in osteoclasts. J. Cell Sci. 108: 2285-2292.

Zhao Q, Wang X, Liu Y, He A, et al. (2010). NFATc1: functions in osteoclasts. Int. J. Biochem. Cell Biol. 42: 576-579. 